\title{
Delayed feedback control of chaos: Bifurcation analysis
}

\author{
A.G. Balanov ${ }^{1}$, N.B. Janson ${ }^{2,1}$, E. Schöll ${ }^{1}$ \\ ${ }^{1}$ Institut für Theoretische Physik, Technische Universität Berlin, Hardenbergstraße 36, D-10623 Berlin, Germany \\ 2 Department of Mathematical Sciences, Loughborough University, Loughborough, Leicestershire, LE11 $3 T U$, UK
}

(Dated: September 7, 2004)

\begin{abstract}
We study the effect of time delayed feedback control in the form proposed by Pyragas on deterministic chaos in the Rössler system. We reveal the general bifurcation diagram in the parameter plane of time delay $\tau$ and feedback strength $K$ which allows one to explain the phenomena that have been discovered in some previous works. We show that the bifurcation diagram has essentially a multi-leaf structure that constitutes multistability: the larger the $\tau$, the larger the number of attractors that can coexist in the phase space. Feedback induces a large variety of regimes non-existent in the original system, among them tori and chaotic attractors born from them. Finally, we estimate how the parameters of delayed feedback influence the periods of limit cycles in the system.
\end{abstract}

PACS numbers: 05.45.-a, 05.45.Gg

\section{INTRODUCTION}

The problem of control of oscillatory behavior is of long standing. Usually, whenever an irregular, unpredictable motion occurs in an engineering or technological device, the need arises to take some action in order to turn it into a predictable one or to stop any oscillations altogether. With the progress of nonlinear dynamics, intelligent chaos control methods have been developed that exploit the existence of unstable periodic orbits(UPOs) embedded into chaotic attractors [1-4]. The general idea is to turn an unstable orbit into a stable one by applying some small force, and a variety of ways to do this have been proposed so far. Among the most prominent ones is the OGY approach [5] that suggests to wait until the phase trajectory reaches a sufficiently small vicinity of the target saddle orbit, and then apply a small feedback force that would push it towards the orbit along its stable manifold. With all the beauty of the idea, this approach requires quite a lot of knowledge about the orbit including its position and stability properties that are not always straightforward to find in a real-life situation. A famous approach that is lacking the above shortcoming but achieves the same goal, was proposed by Pyragas [6]. It uses a delayed feedback in the form of a signal $\mathcal{F}(t)$ that is proportional to the difference between some function $g$ of the current system state $\mathbf{x}(t)$ and of its state $\mathbf{x}(t-\tau)$ some $\tau$ time units ago:

$$
\mathcal{F}(t)=g(\mathbf{x}(t))-g(\mathbf{x}(t-\tau))
$$

multiplied by a feedback strength $K$. If $\tau$ is precisely equal to the period $T$ of the UPO, the orbit may become stable under the appropriate choice of feedback strength $K$. Note that only the stability properties of the orbit are changed, while the orbit itself and its period remain unaltered. The conditions for $K$ for successful control have been thoroughly studied in [7-10]. Namely, it has been shown that if the orbit has an odd number of real Floquet multipliers greater than unity, the delayed feedback can never stabilize it. Also, for the orbits that can be stabilized, there is a finite range of values of $K$ at which the control can be achieved: on the lower boundary which is always larger than zero the stabilized orbit undergoes a period-doubling bifurcation, while on the upper boundary a torus is born from it. Later, this simple approach was extended to multiple time delays $\sum_{n=0}^{\infty} R^{n} \mathcal{F}(t-n \tau)$ with some memory parameter $|R|<1$ [11], which improves the efficiency of control [12]. Both schemes have been applied to a number of real world problems in various areas of physics, chemistry and biology [13-21]. Recent interest has focussed upon the application to spatially extended systems, and a variety of global and local coupling schemes of the control force have been investigated, e.g. [22-26]. Extensions to spatio-temporal filtering have been used to demonstrate that the efficiency can even be increased by several orders of magnitude [27, 28]. However, in this work we will restrict ourselves to the simplest control scheme provided by Eq. (1).

The Pyragas approach is especially appealing for experimentalists, since one does not need to know anything about the target orbit beyond its period $T$. Note that if $\tau$ is equal to $T$ precisely, once the target orbit is reached, the control force vanishes. That is why this type of control is called non-invasive. However, in an experimental situation the dynamical equations describing the given oscillations are typically not known, and the period of the target orbit may be estimated using a kind of spectroscopy approach: one applies the feedback with values of $\tau$ from a substantially large range, and estimates the control force $\mathcal{F}$ for each $\tau$. If $\tau$ is close to the period of the UPO, the absolute value of control force $\mathcal{F}$ averaged over time approaches zero. Of course, due to the finite numerical resolution, the control force will never vanish, and the accuracy of the thus determined value of the period $T$ depends upon the sampling resolution of the $\tau$-range. It has been shown numerically, however, that if $\tau$ is not equal, but close enough to $T$, 
the orbit changes both its shape and period. In [29] a method was proposed to find a better estimate for $T$ from the knowledge of the periods of the stable periodic orbits at two different values of $\tau$. The method works well if the two values of $\tau$, which are chosen by an intial guess, are close enough to $T$, but its accuracy decreases if they are far from $T$. Interestingly, already in [6] it has been reported that delayed feedback can suppress oscillations and also induce multistability for sufficiently large $K$. The existing works on delayed feedback control generally analyze two complementary cases: (i) $K$ is fixed while $\tau$ is changed, or (ii) $\tau=T$ is fixed while $K$ is changed.

Inspite of the large number of works on delayed feedback control that reveal a variety of phenomena in systems being controlled, the general bifurcation scenarios realized under variation of feedback parameters are still unknown even for the simplest low-dimensional chaotic systems and the simplest form of feedback. One of the practical goals of the current work is to assess the accuracy with which it is sufficient to know the target orbit period $T$ to allow for successful control. We would also like to find out how the range of allowed $\tau$ depends on $K$ : we suggest that a suitably chosen $K$ can broaden the allowed $\tau$ range. Exploration of how oscillations can be damped would be potentially useful in engineering applications. For this purpose we study the response of a chaotic system to the delayed feedback with parameters $K$ and $\tau$ varying in quite large ranges. We perform a systematic bifurcation analysis of the system with delay and find the regions of periodic, quasiperiodic and chaotic motion, as well as those where oscillations are absent, and the regions of multistability.

\section{SURVEY OF THE BIFURCATION DIAGRAM}

We use the Rössler model as a paradigmatic chaotic system to which we apply delayed feedback control. It exhibits chaotic oscillations born via a cascade of period-doubling bifurcations:

$$
\begin{aligned}
& \dot{x}=-y-z-K(x(t)-x(t-\tau)) \\
& \dot{y}=x+a y \\
& \dot{z}=b+z(x-\mu),
\end{aligned}
$$

considered at the parameter values $a=0.2, b=0.2, \mu=6.5$. With $K=0$, at these parameters the attractor of the system is represented in Fig. 1 by dots. Unstable periodic orbits with periods $T_{1} \approx 5.91679$ ("period-one orbit") and $T_{2} \approx 11.82814$ ("period-two orbit") that are embedded into the chaotic attractor are shown by grey and black (red and black on-line) lines, respectively. In Fig. 2 we display a bifurcation diagram in terms of the parameters $\tau$ and $K$; the lines $\tau=T_{1}$ and $\tau=T_{2}$ are depicted by vertical dash-dotted lines. Application of the delayed feedback with $\tau=T_{1}$ and $0.24<K<2.3$ stabilizes the period-one orbit, and it becomes the only attractor of the system. In [9] it has been predicted analytically by a linear expansion that control is realized only in a finite range of the values of $K$ : at the lower control boundary the limit cycle should undergo a period-doubling bifurcation, and at the upper boundary a Hopf bifurcation generating a stable or an unstable torus from a limit cycle (Neimark-Sacker bifurcation). Although the linear expansion becomes exact only for a special coupling of the control force in the form of a unity matrix (diagonal coupling), this general bifurcation behavior has been numerically verified in a large number of diverse delayed feedback control systems including spatially extended reaction-diffusion systems [23-25]. The feedback scheme $(2)$ we use here is non-diagonal, but in qualitative agreement with [9], at $K \approx 0.24$ the stable limit cycle undergoes a period-doubling bifurcation, and at $K \approx 2.3$ a Neimark-Sacker bifurcation.

In Fig. 2 the hatched area touching the coordinate axes approximately outlines the region of chaos born through a cascade of period-doubling bifurcations of the main period-one cycle that is born via a Hopf bifurcation at the borderlines of the closed loops marked as black areas. In other words, the delayed feedback leads to a cascade of inverse period-doubling bifurcations of chaos as $\tau$ is increased from zero for a visible range of fixed $K$, or as $K$ is increased from zero for most values of $\tau$ from the range investigated.

It was first mentioned in [6], and later illustrated in more detail, e.g., in [30, 31] that delayed feedback can not only stabilize the originally unstable periodic orbit, but also, with the appropriate choice of $\tau$ and $K$, induce the inverse Hopf bifurcation of this orbit, thus suppressing oscillations in the system altogether. The same phenomenon occurs in Eqs. (2). In Fig. 2 black shaded loops show the stability domains of the fixed point close to the origin of phase space, which for $K=0$ and the parameters $a, b, \mu$ chosen is an unstable saddle-focus with approximate eigenvalues $\lambda_{1,2}=a / 2 \pm i$ and $\lambda_{3}=-\mu$. That is, inside the black regions no oscillations occur. Note that the largest such region is situated to the left of, and quite close to, the line $\tau=T$. The loops containing the stable fixed point form a self-similar structure in the $(K, \tau)$ parameter plane, and decrease in size as $\tau$ grows, in full agreement with [30, 31].

The thick black solid lines in Fig. 2 forming loops are the lines of Hopf bifurcation at which a periodic orbit is born. The bifurcation diagram has a multi-leaf structure, and the borderlines of different leaves are formed by the segments of Hopf bifurcation lines according to the rule illustrated by Fig. 3. Each leaf is defined as being the one on which the same periodic orbit of period one and all attractors born from it exist and undergo bifurcations. In Fig. 


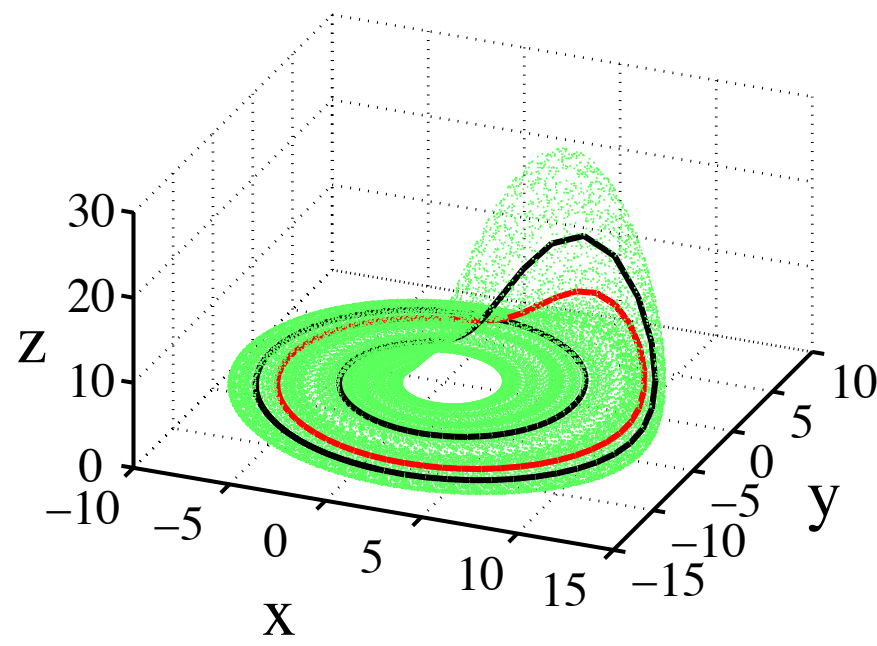

FIG. 1: Dots: chaotic attractor of Rössler system Eq. (2) without delayed feedback $(K=0)$, grey line (red on-line): period-one unstable periodic orbit, black line (black on-line): period-two unstable periodic orbit.

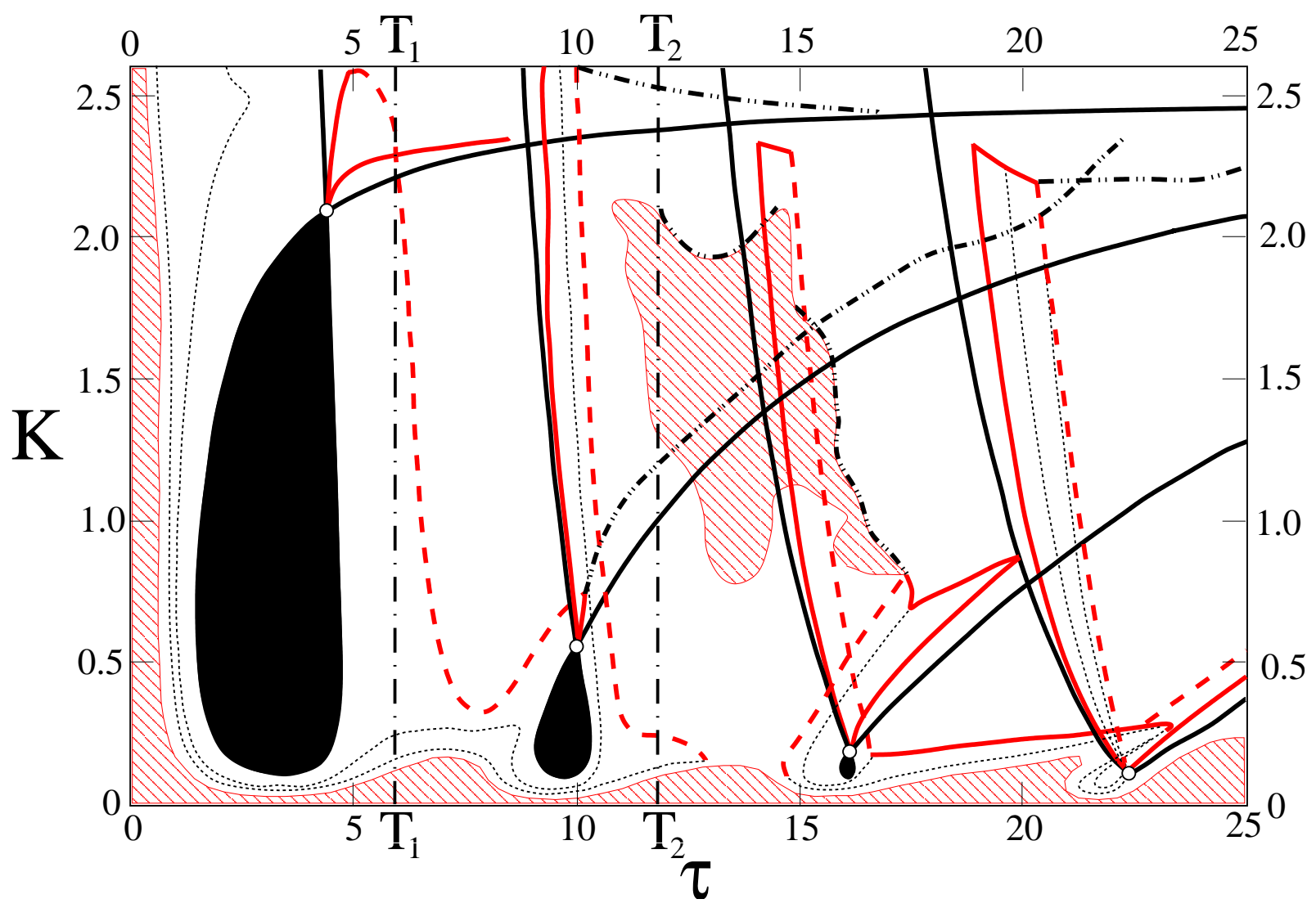

FIG. 2: Bifurcation diagram of the Rössler system with delayed feedback in the plane of feedback parameters: time delay $\tau$ and feedback strength $K$. Bifurcation lines of all leaves are shown. A scheme of the leaf structure is given in Fig. 3. For bifurcations on separate leaves see Fig. 5.

Black areas mark the existence of a stable fixed point, which never coexists with any other attractors. Black solid lines mark Hopf bifurcations of the fixed point, at which a periodic orbit (stable or unstable) is born. White circles mark points of self-intersection of Hopf bifurcation line. On grey (red on-line) solid lines an unstable limit cycle acquires stability via a subcritical Neimark-Sacker bifurcation. On grey (red on-line) dashed lines a stable torus is born from the stable limit cycle via a supercritical Neimark-Sacker bifurcation. Dotted lines show period-doubling bifurcations of stable limit cycles. On dash-double-dotted lines the attractors undergo crises and cease to exist. Hatched areas denote chaos born as a result of period-doubling bifurcations or of smoothness loss by the invariant torus. 


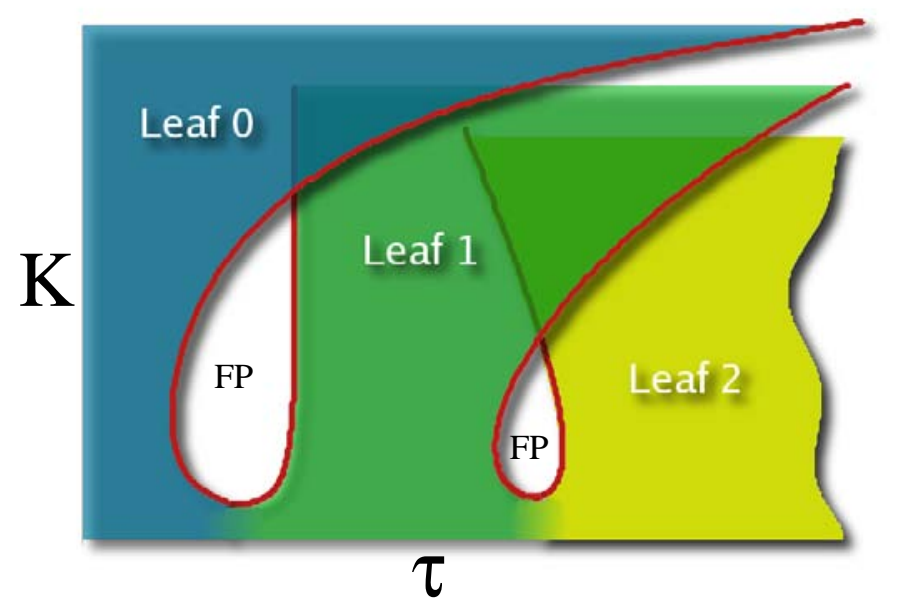

FIG. 3: Sketch of multiple leaf structure of bifurcation diagram of system (2) on the parameter plane $(K, \tau)$. Letters "FP" mark the stability regions for the only fixed point of the system 2 .

4 it is illustrated how a leaf is determined for a particular value of $K$. The sizes of period-one orbits, quantified by the differences $x_{\max }-x_{\min }$ of the $x$ variable, are shown $v s \tau$ for three values of $K$ indicated in the figure. Each orbit has zero size when it is born (dies) via Hopf bifurcation on the left-hand (right-hand) border of the leaf. The range of the orbit existence defines the leaf. Shaded areas mark the stability of each orbit. The particular periodic orbit and associated attractors do not exist outside their respective leaf.

Although all relevant bifurcation lines are shown in Fig. 2, the complexity of the diagram may make it difficult to associate each line with the appropriate leaf. For easier visualization we cut out the different leaves and show them separately in Fig. 5, where shaded areas do not belong to the leaf considered. Since all leaves merge for small $K$, the cutting of separate leaves is done along the arbitrarily selected vertical lines on the diagram that pass through the lowest points of the loops of Hopf bifurcation lines.

From Fig. 3 it is clear that for large $K$ different leaves overlap, and multistability occurs. For the clarity of description it is convenient to number the leaves. We assign number 0 to the leaf that is not complete, i.e. whose left-hand boundary is formed by the axis $\tau=0$. Leaf 0 has the simplest structure. Namely, on the line of Hopf bifurcation a periodic orbit of period one is born from the fixed point. It is born stable in the lower part of the diagram and unstable above the point at which the Hopf bifurcation line intersects itself (white circle in Figs. 2, 5). From the latter point a line of subcritical Neimark-Sacker bifurcation emerges (shown by a solid grey line in Figs. 2 and 5, leaf 0): as it is crossed from below to above, the unstable periodic orbit acquires stability. With this, as this line is crossed from above to below, the limit cycle looses its stability, but no stable torus is born from it. As the point in the parameter plane moves towards the axes $\tau=0$ and/or $K=0$, the stable limit cycle undergoes a period-doubling bifurcation. A cascade of period-doubling bifurcations leads to the birth of a chaotic attractor which at $\tau=0$ or $K=0$ becomes the attractor of the system (2) without feedback shown in Fig. 1 by dots. An important feature of this leaf is that at large $K$ on the dash-double-dotted line the limit cycle vanishes through a crisis, and the trajectory abruptly goes to infinity. Thus, above the crisis line there are no attracting sets on this leaf.

To all other leaves we assign positive integer numbers 1, 2, 3, 4, etc. Although different leaves have generally different structures, they possess some common features. The periodic orbit is born stable below the point at which the Hopf bifurcation line intersects itself (white circles in Figs. 2 and 5), and unstable above this point. From the latter point a subcritical Neimark-Sacker bifurcation line emerges (grey solid line in Figs. 2 and 5), that being crossed in the direction towards the leaf centre renders the originally unstable periodic orbit stable. As one moves deeper inside the leaf, the stable periodic orbit undergoes one of two bifurcations: supercritical Hopf bifurcation that leads to the birth of a stable two-dimensional torus (grey dashed line in Figs. 2 and 5), or period-doubling bifurcation (dotted line). It may happen that the line of subcritical Neimark-Sacker bifurcation is continued as, or crossed by, a line of supercritical Neimark-Sacker bifurcation, as happens close to both borders of leaves 1 and 3, to the right-hand border of leaf 2 , and to the left-hand border of leaf 4 .

While bifurcations of fixed points and periodic orbits were detected by means of continuation tools [32], transformations of objects with higher dimensions were detected by observation of their Poincaré maps that were calculated from the condition $\dot{x}=0$. The structure of the Rössler equations is such that all trajectories should cross the plane 

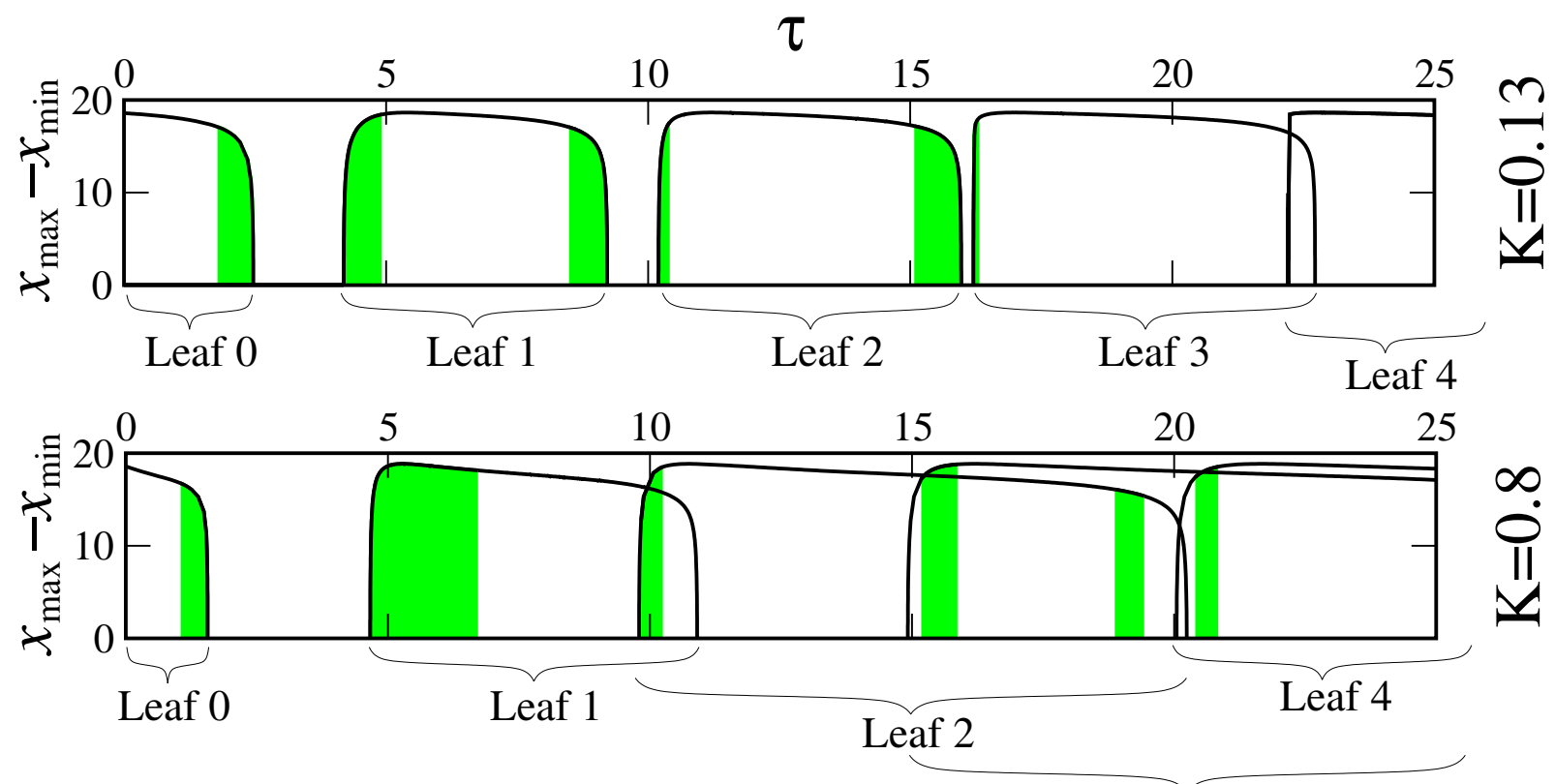

Leaf 3

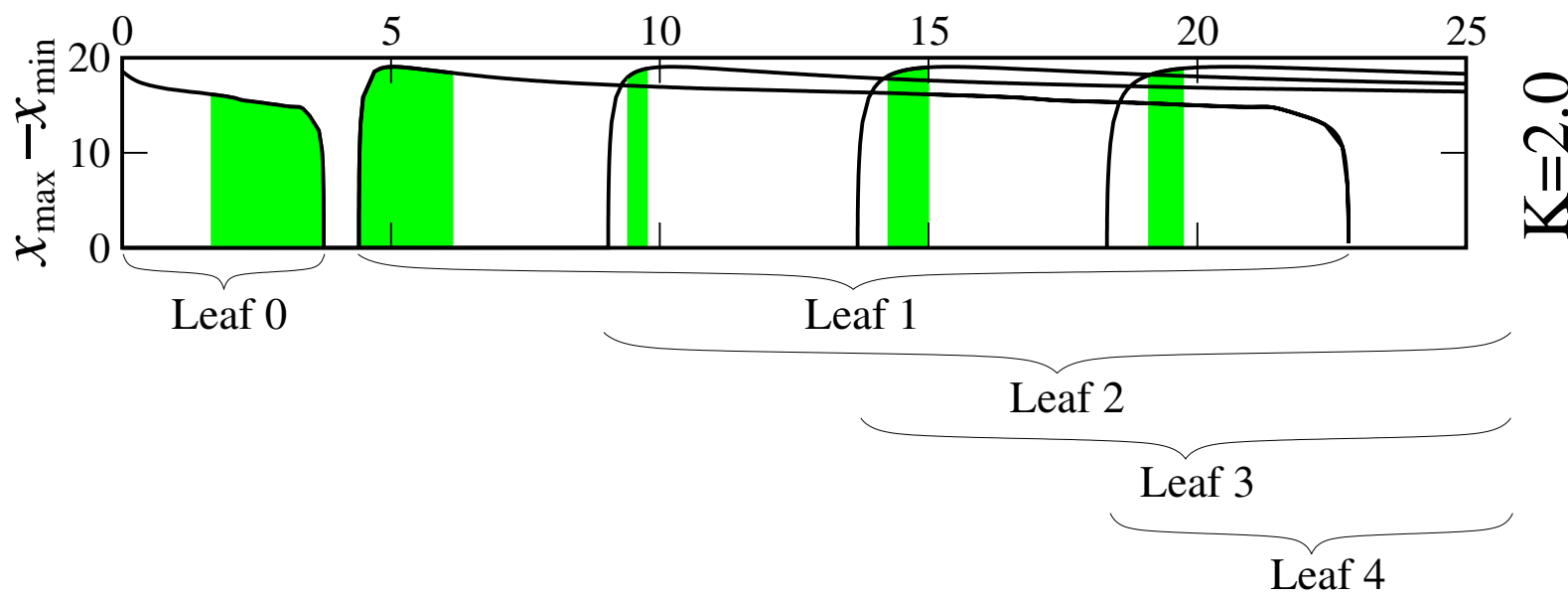

FIG. 4: Illustration of how the leaves of the bifurcation diagram are formed. In each plot the spread of the $x$-variable $x_{m a x}-x_{\min }$ as a measure of the size of the period-one orbit vs $\tau$ is given for a value of feedback strength $K$ that is indicated to the right of the plot. The width of the leaf in $\tau$ is defined by the range of $\tau$ where the particular orbit has a non-zero size. Inside the shaded areas the orbit is stable.

defined by the above equation. In uncertain cases we also calculated Fourier power spectral densities from the variable $x(t)$.

Generally, as one moves deeper inside the leaf, a stable two-dimensional torus appears, being born from a period-one limit cycle as in leaves 1 and 3 , or from a period-two cycle as in leaf 2 , or from a period-four limit cycle as in leaf 4 . Hence, large portions in the centers of all leaves are the domains of existence of a two-dimensional torus. With this, as parameters are changed inside the latter domains, the two-dimensional torus may undergo various bifurcations, e.g., another Neimark-Sacker bifurcation leading to the birth of a three-dimensional torus, torus doubling (sometimes a cascade of torus doublings), and merging of torus bands (e.g for tori born from a period-two or period-four orbit). A torus can become resonant, i.e. visible in the Poincaré map as the appearance of a discrete number of points in place of a closed circle. However, most commonly, the torus loses its smoothness, breaks down, and turns into a chaotic attractor. Because there are currently no numerical methods available that would allow continuation of objects more complex than periodic orbits, all bifurcations of tori were detected by visual inspection of Poincaré maps accompanied by calculation of Fourier power spectral densities where necessary. An example of this is given in Fig. 6 where the transition from a smooth torus to chaos is illustrated for leaf 2 . We fix $\tau$ at 13.5 and gradually increase $K$ from 0.74 to 

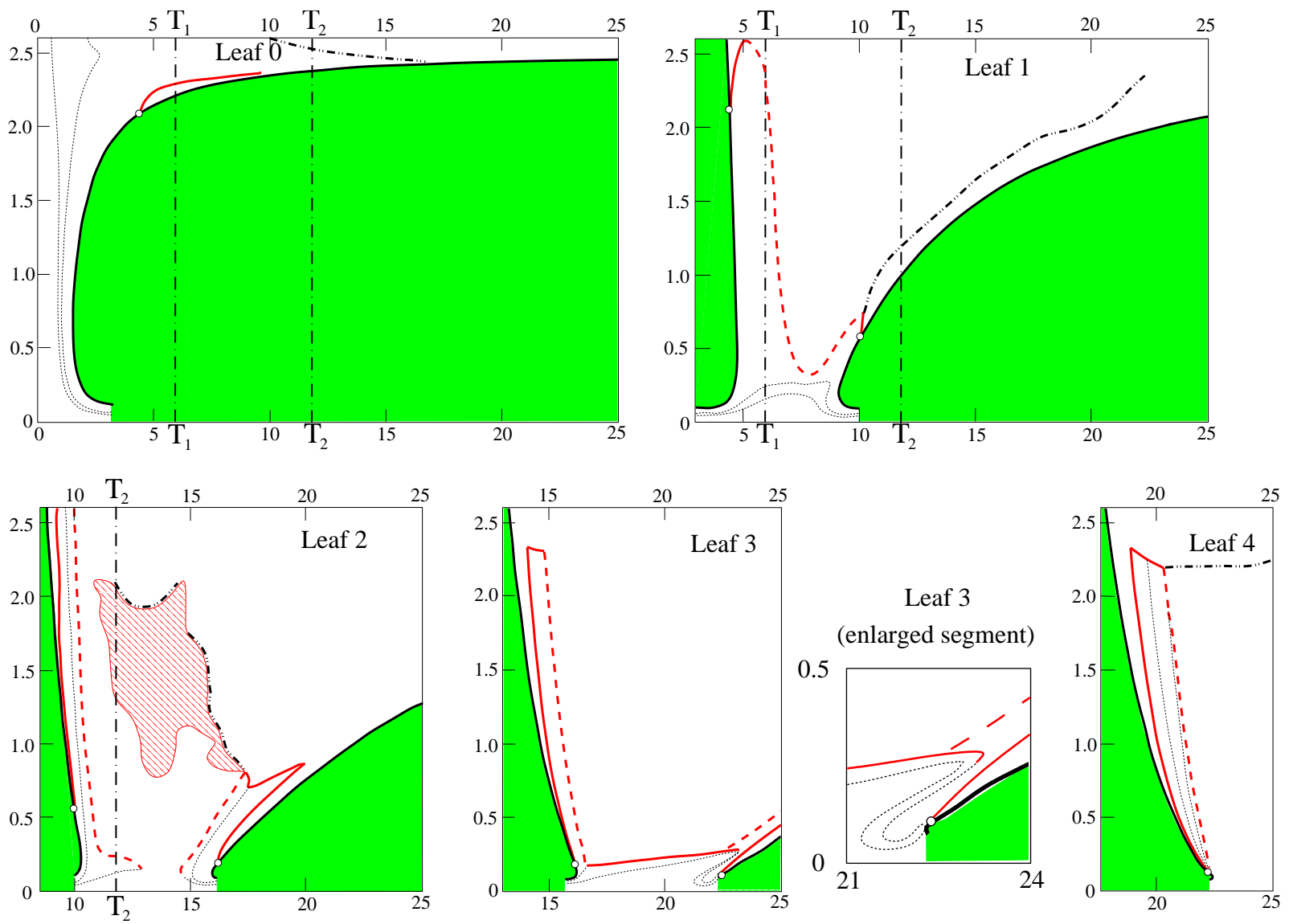

FIG. 5: Separate leaves of the bifurcation diagram of the Rössler system with delayed feedback (shown in full in Fig. 2). Shaded grey areas do not belong to the respective leaf. Lines are denoted in the same way as in Fig. 2.
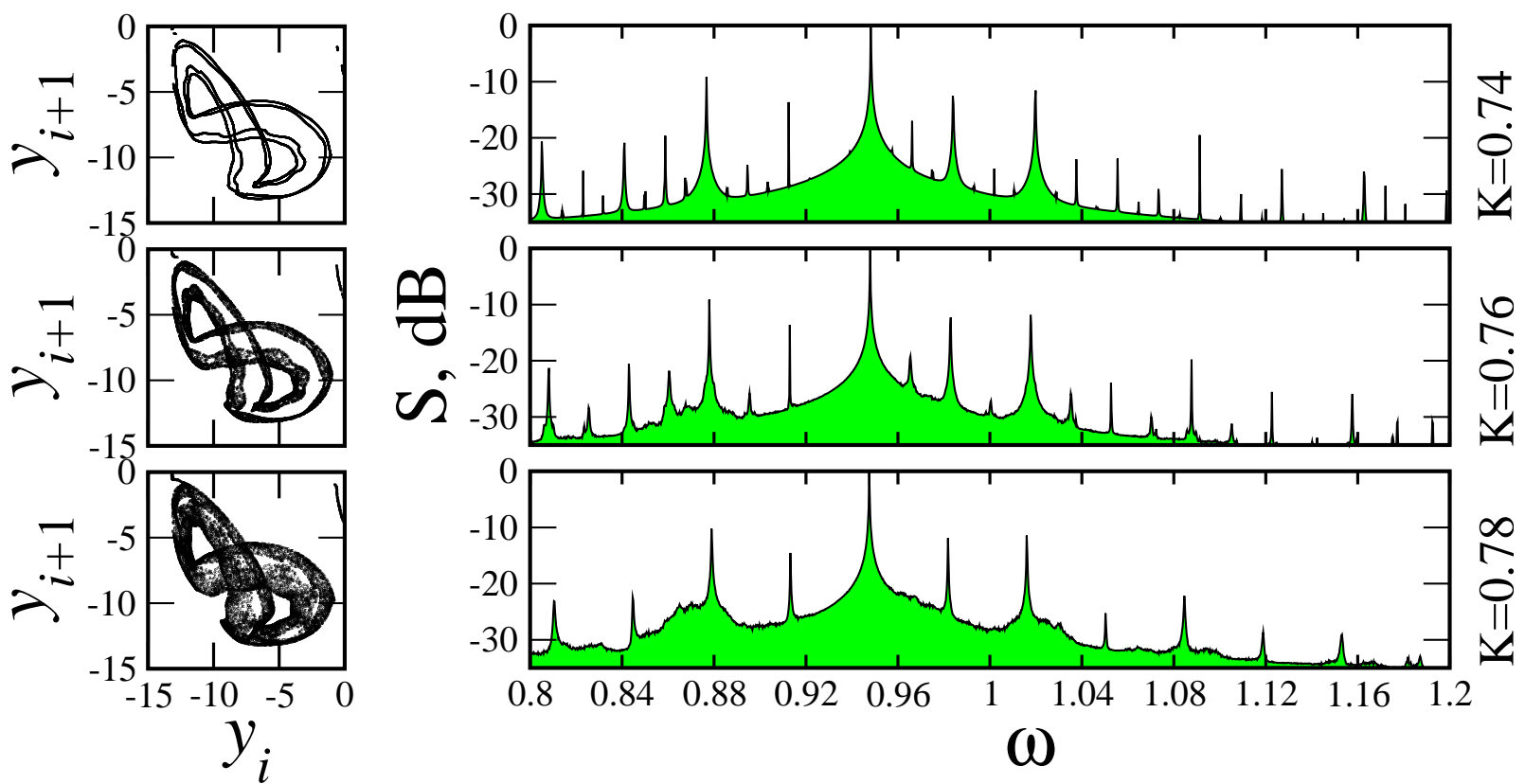

FIG. 6: Illustration of torus breakdown as a transition to chaos in the Rössler system with delayed feedback on leaf 2 of the bifurcation diagram (Fig. 5). Poincaré maps of attractors and their respective Fourier power spectral density $S$ calculated from $x(t)$ are given for three values of $K$ at $\tau=13.5$. Each panel illustrates a particular value of $K$. 

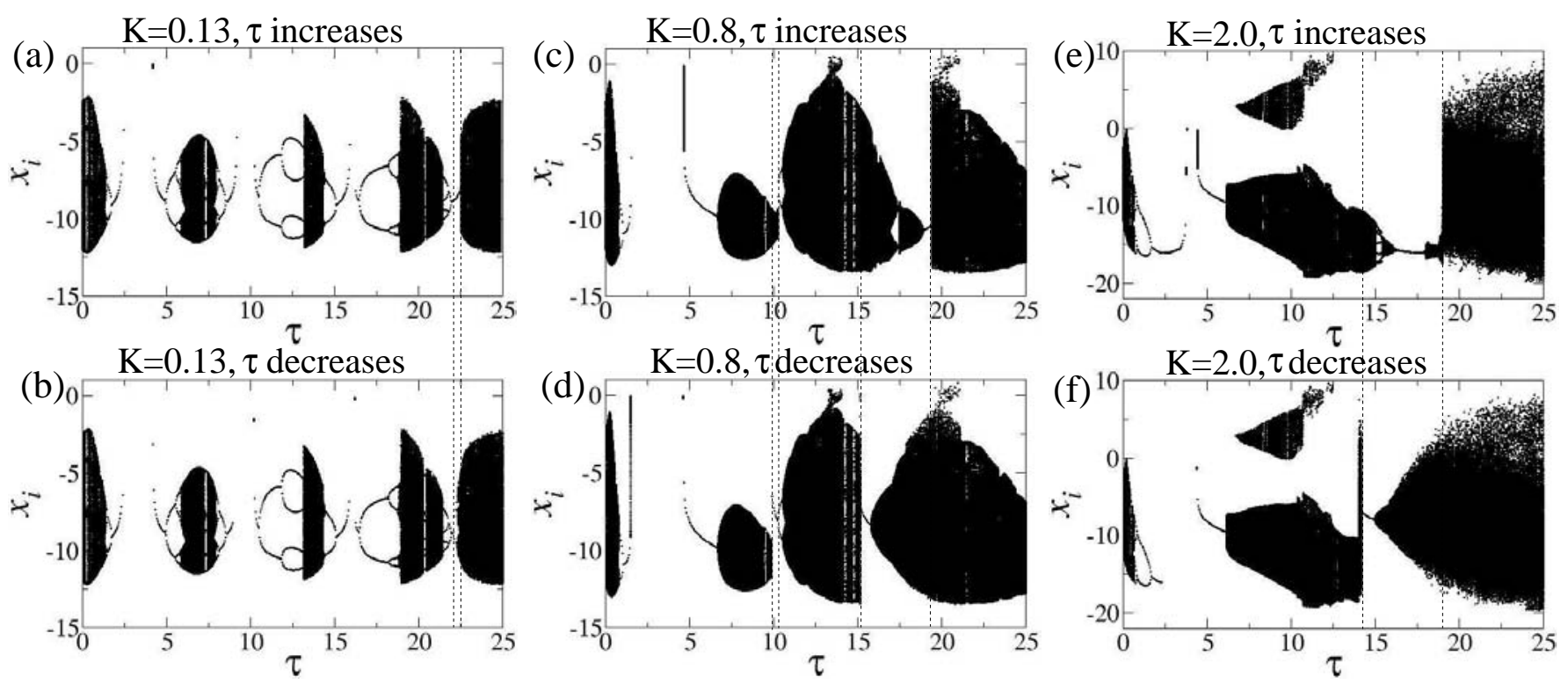

FIG. 7: One-parameter bifurcation diagrams of system (2), illustration of multistability: for each fixed $K, \tau$ is first increased from zero to 25 and then decreased from 25 to zero. (a), (b) $\mathrm{K}=0.13$ (c), (d) $\mathrm{K}=0.8$, (e), (f) $\mathrm{K}=2.0$.

0.78. In Fig. 6 Poincaré sections are shown for three values of $K$ together with the Fourier power spectral density $S$. One can see that the two-dimensional torus visualized by a period-four invariant closed curve in the Poincaré section breaks down giving birth to a chaotic attracting set. This is confirmed by the power spectrum $S$, which at $K=0.74$ is discrete within the numerical accuracy, at $K=0.76$ becomes enriched by discrete components and at $K=0.78$ becomes continuous. The transition from a discrete to continuous spectrum is clearly visible as small spectral peaks are widened substantially.

The lines of bifurcations of tori are quite dense in the parameter plane, and it is impossible to reveal them all within reasonable computational effort. For this reason, in this work we only outline the largest domain of chaos born via torus breakdown that was found on leaf 2 (hatched area in Fig. 5). On leaf 1 the stable two-dimensional torus undergoes bifurcations only approximately in the upper fifth of the diagram (not illustrated). On other leaves the chaotic domains were relatively narrow.

Importantly, although each leaf is defined by the existence of a particular periodic orbit, the attracting sets do not exist in the whole area of the leaf. Obviously, there are no attractors in the areas between Hopf and subcritical Neimark bifurcations (solid black and solid grey lines in Fig. 5), because the just born periodic orbit is unstable there and does not create any other attractors yet. With this, the attracting sets that are born through local bifurcations of the periodic orbit can undergo crises as the parameters are changed, and the phase trajectory jumps to one of the coexisting attractors. The lines of crises are shown by dash-double-dotted lines in Figs. 2 and 5.

In particular, the line of crisis of the two-dimensional torus of leaf 1 roughly follows the Hopf bifurcation line: as one approaches the crisis line from the left, the torus diameter becomes very small as if the torus is about to undergo an inverse supercritical Neimark-Sacker bifurcation. But the latter does not happen and instead the torus disappears abruptly.

In leaf 2 the chaotic attractor vanishes through a crisis on two lines that we managed to reveal by numerical simulation. As the line that is oriented mostly vertically is crossed, the phase trajectory jumps on the attractor of leaf 3. This line was not extended above the upper point shown since there was no crisis detected for the slightly larger values of $K$ checked. As the upper line is crossed, the phase trajectory jumps on the attractors of leaf 1 . This line was also not extended beyond the limits shown because of the numerical uncertainties that occurred in the regions investigated.

Also, attractors of all leaves undergo some crisis at $K$ close to, or immediately above, the upper line of the Hopf bifurcation (right-hand border of leaf 0 ) at $\tau$ larger than 10. This crisis is detected in numerical simulation as follows: starting from an attractor of a certain leaf, as one increases $K$, this attractor abruptly vanishes and the trajectory jumps to the attractor of the preceding leaf. As $K$ is increased further, the current attractor vanishes again. This is repeated until leaf 0 is reached. As $K$ is increased further, the attractor of leaf 0 vanishes and the trajectory goes to infinity.

In order to reconstruct the actual bifurcation diagram, one can cut out separate leaves from Fig. 5 leaving out the 


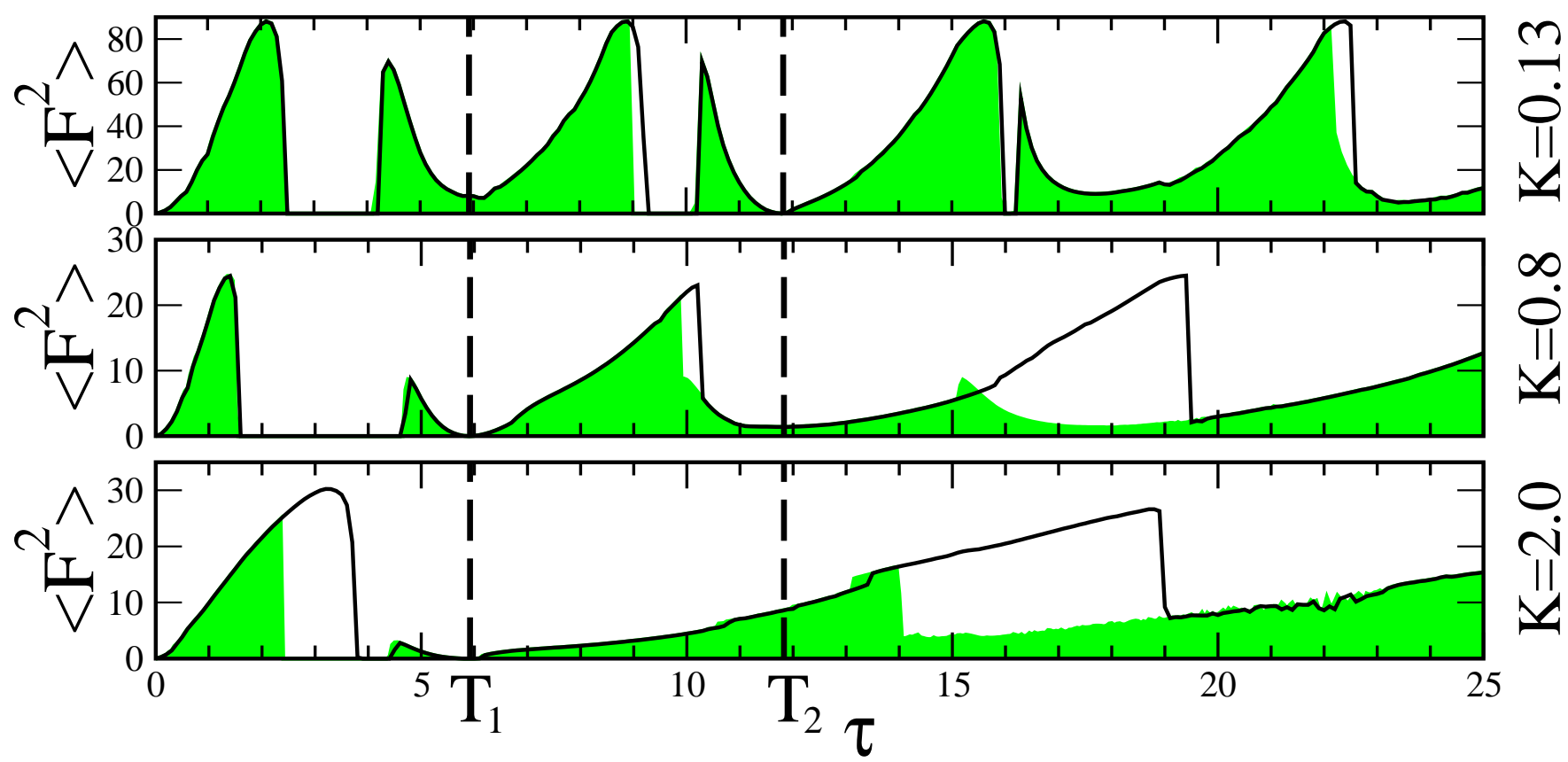

FIG. 8: Variance of control force $F$ defined by Eq. (3) vs $\tau$ for three different value of $K$ : (a), (b) $\mathrm{K}=0.13$ (c), (d) K=0.8, (e), (f) $\mathrm{K}=2.0$. For each fixed value of $K, \tau$ is first increased from zero to 25 (black line) and then decreased from 25 to zero (shaded area). Multistability is clearly visible.

shaded areas, and glue leaves with consecutive numbers together: leaf 1 should be glued to leaf 0 from the right, leaf 2 to leaf 1 , etc.

In order to illustrate multistability and the change of regimes along the parameter plane, we plot three one-parameter bifurcation diagrams along the lines $K=0.13, K=0.8, K=2.0$. Points of the projections of the Poincaré map onto variable $x$ are plotted versus $\tau$, as $\tau$ is first increased from 0 to 25 (upper panels in Fig. 7), and then decreased from 25 to 0 (lower panels in Fig. 7). Vertical dotted lines are put at the values of $\tau$ at which jumps between different leaves occur. The same line embraces both the upper and the lower plot for the same value of $K$ in order to emphasize that jumps occur at different values of $\tau$ depending on whether $\tau$ is being increased or decreased. The larger the value of $K$ is, the larger the gaps in $\tau$ between these jumps are.

Also, for potential applications it is interesting how the magnitude of the control force depends on the feedback parameters. We define the control force $\mathcal{F}=F$ as

$$
F(t)=x(t)-x(t-\tau)
$$

and calculate the mean value $\langle F\rangle$ and the variance $\left\langle F^{2}\right\rangle-\langle F\rangle^{2}$ of this variable through averaging over the observation time. The mean value of the control force $\langle F\rangle$ is zero within numerical accuracy. The plots of the variance $\left\langle F^{2}\right\rangle$ of the control force vs $\tau$ for three values of $K$ are shown in Fig. 8: black lines show $\left\langle F^{2}\right\rangle$ calculated as $\tau$ was increased from zero to 25 , and shaded areas show $\left\langle F^{2}\right\rangle$ calculated as $\tau$ was decreased from 25 to zero. Hysteresis is clearly visible in these plots, and is due to overlapping of different leaves of the bifurcation diagram. Segments of finite length where $\left\langle F^{2}\right\rangle$ is zero represent the stability domains of the fixed point. Points at which $\left\langle F^{2}\right\rangle$ reaches (or tends to) zero are those at which $\tau$ coincides with some accuracy with the periods of period-one (at $K=0.8$ and $K=2.0$ ) or period-two (at $K=0.13$ ) unstable periodic orbits in the original system (2) that are marked by vertical dash-dotted lines. One can see that the control force is small in quite large vicinities of these points. Note that with those three arbitrarily selected values of $K$ we were unable to find orbits whose stability domains in our bifurcation diagram are very small and are not shown, like period-three and period-four orbits. This spectroscopy approach has been proposed in [6] for the use in experiments in order to estimate the periods of unstable obits. However, Fig. 8 illustrates that in the presence of multistability that is inevitably induced by delayed feedback, an arbitrary selection of feedback strength $K$ does not allow one to reveal all orbits. To reveal all orbits, one should in fact scan the system in a wide range of $K$.

We note that although one can in principle stabilize a period-three periodic orbit whose period is $T_{3} \approx 18$ and thus falls within the $\tau$ limits we consider, the range of its stability over both $K$ and $\tau$ is very small, and we do not show it in this work. 


\section{ESTIMATION OF PERIODS OF LIMIT CYCLES}

In practice it is often desirable to know in advance the outcome of application of delayed feedback to the particular system. That includes the knowledge of the period of the resulting stable orbit. In order to estimate how the parameters $K$ and $\tau$ of delayed feedback influence the period of this orbit, we use an approach suggested in [29], where the following relationship for the resulting limit cycle of period $\Theta(K, \tau)$ in the system with delayed feedback has been derived:

$$
\left.\kappa \partial_{\tau} \Theta\right|_{\tau=T}+\left.T K \partial_{\tau}(\tau / \Theta)\right|_{\tau=T}=0
$$

Here the operator $\partial_{\tau}$ denotes the derivative with respect to $\tau$. $\kappa$ is a system parameter characterizing the effect of the control force on the system dynamics, which does not depend on $K$ and $\tau$ for a given controlled orbit. $T$ denotes the period of the unperturbed orbit at $K=0$. Following the derivation in [29], one can show that all statements obtained there for $\tau=T$ remain valid also for $\tau=n T$ where $n$ is a non-negative integer. The argument is that if the initial conditions are set precisely on the orbit with period $T$, the control force $F(t)=x(t)-x(t-\tau)$ is zero for any $\tau=n T$. Hence we can rewrite Eq.(4) as

$$
\left.\kappa \partial_{\tau} \Theta\right|_{\tau=n T}+\left.T K \partial_{\tau}(\tau / \Theta)\right|_{\tau=n T}=0, n=0,1,2 \ldots
$$

or as

$$
\begin{aligned}
\left.\kappa \partial_{\tau} \Theta\right|_{\tau=n T} & +\left.T K \frac{1}{\Theta}\right|_{\tau=n T} \\
& -\left.T K \frac{\tau}{\Theta^{2}} \partial_{\tau} \Theta\right|_{\tau=n T}=0, n=0,1,2 \ldots .
\end{aligned}
$$

Taking into account that $\Theta(K, n T)=T$, we find

$$
\left.\kappa \partial_{\tau} \Theta\right|_{\tau=n T}+K-\left.n K \partial_{\tau} \Theta\right|_{\tau=n T}=0, n=0,1,2 \ldots,
$$

and finally obtain the derivative of the period $\Theta$ with respect to time delay $\tau$

$$
\left.\partial_{\tau} \Theta\right|_{\tau=n T}=\frac{K}{n K-\kappa}, n=0,1,2 \ldots
$$

Expansion of $\Theta$ in a Taylor series yields

$$
\begin{array}{r}
\Theta(K, \tau)=T+\left.\partial_{\tau} \Theta\right|_{\tau=n T}(\tau-n T)+\mathcal{O}\left((\tau-n T)^{2}\right), \\
n=0,1, \ldots
\end{array}
$$

In this relationship we keep only the linear term and substitute Eq. (7) to obtain an approximate formula for the period of the delay-influenced orbit:

$$
\Theta(K, \tau) \approx T+\frac{K}{n K-\kappa}(\tau-n T), n=0,1,2 \ldots
$$

Thus, in order to find the period of the controlled orbit, besides the control parameters $K$ and $\tau$ one needs to know also the period of the unperturbed orbit $T$ and the parameter $\kappa$ that characterises the integral effect of the feedback on the system that does not depend on $K$ and $\tau$ for the given orbit. If $T$ could be somehow revealed from the response of the system to delayed feedback over a wide range of values of $\tau$ [6], the calculation of $\kappa$ appears to be a difficult problem [29]. Moreover, for each new orbit this parameter will be different. However, the situation is a bit simplified by the observation that if an orbit of period $T$ is characterized by a parameter $\kappa=\kappa_{1}$, then the parameter for the orbit of period $m T, m=1,2, \ldots$, will be $\kappa_{m}=\kappa_{1} / m$. This feature of $\kappa$ follows from Eq.(4) and the definition of the function $\Theta(K, \tau)$, according to which $\Theta(K, T)=\Theta(K, m T)=T$. Using the above statement allows one to generalize the formula (9) to the case of period- $m$ orbits:

$$
\begin{array}{r}
\Theta(K, \tau) \approx m T+\frac{K}{n K-\kappa / m}(\tau-n m T), \\
n=0,1,2, \ldots, m=1,2, \ldots,
\end{array}
$$

where $T$ and $\kappa$ correspond to the period-one orbit. We note that the orbit with period precisely equal to $m T$ might not exist in the original system. However, it is very likely that some orbits with periods close to $m T$ do exist, and thus we expect that the derived relationship holds approximately. 
(a)

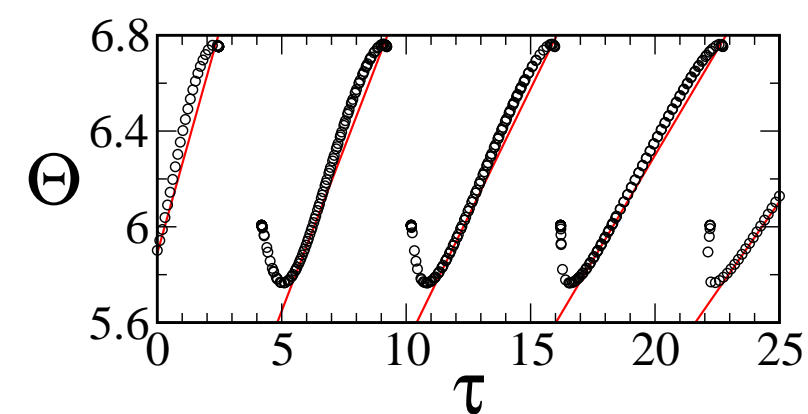

(b)

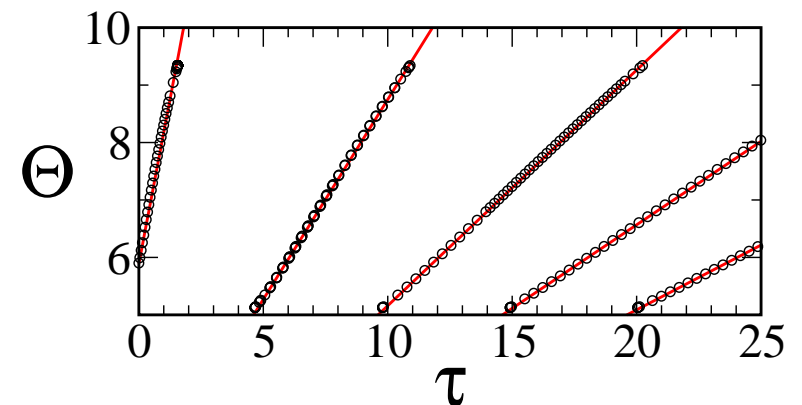

(c)

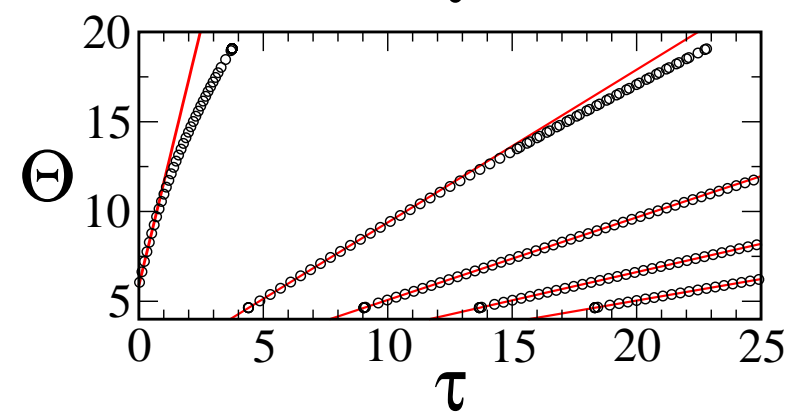

FIG. 9: Period $\Theta$ of period-one orbit (stable or unstable) vs time delay $\tau$ for (a) $K=0.13$; (b) $K=0.8$; (c) $K=2.0$. Black circles denote the period $\Theta$ calculated numerically, the five grey (red on-line) solid lines show the estimates of $\Theta$ using Eq. (9) for $n=0,1,2,3,4$ (from left to right).

To check the formula (10) we numericaly calculate periods of different orbits for a variety of values of $K$ and $\tau$. First, we estimate the period $T$ of the period-one orbit without control. In our case it appears to be $T \approx 5.91679$. In Fig. 9 the dependences of periods of period-one orbits (that can be stable or unstable) on time delay $\tau$ are presented for three different values of $K$. Black circles show the periods estimated numerically for period-one orbits from different leaves of the bifurcation diagram (Figs. 2 and 5). Periods calculated using formula (10) are denoted by grey (red on-line) lines. With this, different lines were found for different values of $n=0,1,2,3,4$. As it was mentioned above, the parameter $\kappa$ is not easy to find using the analytic expressions provided in [29]. Therefore, we estimate $\kappa$ for $K=0.8$ by substituting the values of periods $\Theta(\kappa, \tau)$ found numerically for two different values of $\tau$ into Eq. (9), and resolving the system of two equations with respect to $\kappa$. As a result we obtain $\kappa \approx 0.35$. As one can see from Fig. 9, the values of the periods $\Theta$ calculated numerically are in a good agreement with those estimated by formula (10) within a certain range of parameter $\tau$. The evolution of the periods of period- $m$ orbits with the change of $\tau$ is illustrated in Fig. 10 where the estimates of the periods $\Theta$ of period-two and period-four orbits using Eq. (10) are compared with their true values.

Note that in Eqs. (5)-(10) $n$ is exactly the number of the leaf of the general bifurcation diagram of the system being controlled that was described in Sec. II. Thus, Eq. (10) provides one with the way to classify the periodic orbits in the systems subject to delayed feedback. Namely, index $n$ defines the leaf on which the orbit exists, and $m$ describes the number of loops the orbit makes before it closes. From the practical viewpoint this means that in an experiment it is enough to measure the orbit period for two values of $\tau$ at the same values of $K$ in order to estimate the period of any orbit with period being multiple of $T$. 
(a)

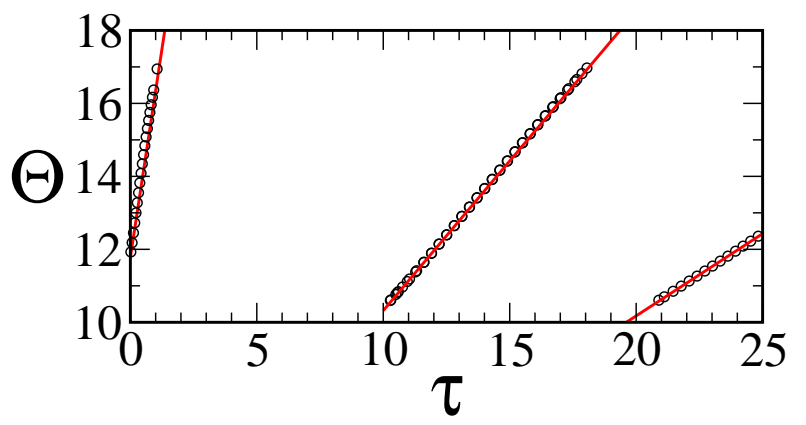

(b)

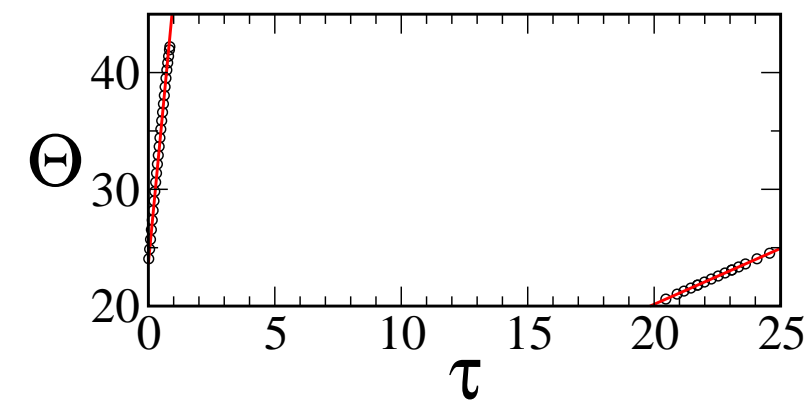

FIG. 10: Period $\Theta$ (a) of period-two orbit for $K=0.8$ and (b) of period-four orbit for $K=2.0$ vs time delay $\tau$. Black circles denote periods calculated numerically, grey (red on-line) lines show estimates with the formula (10) at $m=2$ and $n=0,1,2$ (from left to right) for period-two cycles, and at $m=4$ and $n=0,1$ for period-four cycles.

\section{DISCUSSION AND CONCLUSIONS}

We have revealed the general structure of the bifurcation diagram of a system with chaos born through a cascade of period-doubling bifurcations that is subjected to a delayed feedback control in the form originally proposed by Pyragas [6]. The bifurcation diagram is explored in dependence upon the two feedback parameters: feedback strength $K$ and feedback delay $\tau$. The range of $\tau$ is chosen to be between 0 (absence of feedback) and 25 (slightly more than four times the period of the period-one UPO in the original chaotic system). $K$ is investigated in the range $0<K<2.65$ : within this range at least one attractor of the system with feedback exists for all values of $\tau$ considered. The selected range of feedback parameters has allowed us to discover a multi-leaf self-similar structure of the bifurcation diagram and to reveal some regularities in the structures of different leaves. Each leaf is defined by the existence of a particular periodic orbit and the limit sets born as a result of its bifurcations. The general structure of the leaves and typical bifurcation lines have been worked out. Several regions of chaos were revealed and the largest regions of chaos were shown in Fig. 2.

On the lines $\tau=T_{1}$ and $\tau=T_{2}$ the regions of stability of the period-one and period-two orbits are in good agreement with the analytical predictions of previous works. Relatively small vicinities of these lines are well described by analytical relations as a function of $\tau$ derived in [29] and generalized in the present work for the case of period- $m$ orbits lying on different leaves of the bifurcation diagram. Namely, the actual periods of the period- $m$ orbits stabilized by time delayed feedback depend almost linearly on $\tau$ within a certain range of $\tau$. The areas of the parameter plane where no stable oscillations occur, i.e. where an unstable fixed point is stabilized, are found to be in a good agreement with the results of earlier works.

The results of this work allow us to draw the following conclusions:

(i) The larger the period of the orbit, the smaller is the domain of its stabilization in $(K, \tau)$ parameter space.

(ii) The two largest domains of existence in the parameter plane are the one of a period-one stable orbit, and the one of the stable fixed point which is located inside the former. By selecting $\tau$ only $16 \%$ less than the period of this orbit, one hits the domain where solely a fixed point is stable. As $\tau$ is increased, the stability domains of the fixed point shrink quickly, i.e. the probability of suppressing oscillations at an arbitrarily selected value of $K$ drops dramatically.

(iii) There are several domains of stability of the period-one limit cycle: not only in the vicinity of $\tau=T_{1}$, but also for larger values of $\tau$. However, the stability domain around $\tau=T_{1}$ is the widest in dependence on $\tau$.

(iv) There are several domains of stability of the period-two cycle: not only in the vicinity of $\tau=T_{2}$, but also for smaller or larger values of $\tau$. 
(v) By appropriately choosing the feedback parameters, one can stabilize the period-one orbit with a relatively small control force that will vanish completely on the line $\tau=T_{1}$.

(vi) Increase of both $\tau$ and $K$ leads to severe multistability. Attracting sets of different leaves change quickly with variation of control parameters, so for an arbitrarily chosen pair $(\tau, K)$ it is practically impossible to predict the observed behavior of the system.

\section{ACKNOWLEDGEMENTS}

This work was supported by DFG in the framework of Sfb 555.

[1] H. G. Schuster, Handbook of chaos control (Wiley-VCH, Weinheim, 1999).

[2] S. Boccaletti, C. Grebogi, Y.-C. Lai, H. Mancini, and D. Maza, Phys. Rep. 329, 103 (2000)

[3] D.J. Gauthier, Am. J. Phys. 71, 750 (2003).

[4] W. Just, H. Benner, and E. Schöll, in Advances in Solid State Phyics, edited by B. Kramer (Springer, Berlin, 2003), pp. 589-603.

[5] E. Ott, C. Grebogi, and J. A. Yorke, Phys. Rev. Lett. 64, 1196 (1990).

[6] K. Pyragas, Phys. Lett. A 170, 421 (1992).

[7] M. E. Bleich and J. E. S. Socolar, Phys. Lett. A 210, 87 (1996).

[8] W. Just, E. Reibold, H. Benner, K. Kacperski, P. Fronczak, J. Holyst, Physics Letters A 254, 158-164 (1999).

[9] W. Just, T. Bernard, M. Ostheimer, E. Reibold, and H. Benner, Phys. Rev. Lett. 78, 203-206 (1997).

[10] H. Nakajima, Phys. Lett. A 232, 207-210 (1997).

[11] J. E. S. Socolar, D. W. Sukow, and D. J. Gauthier, Phys. Rev. E 50, 3245 (1994).

[12] K. Pyragas, Phys. Lett. A 206 (1995) 323.

[13] S. Bielawski, D. Derozier, and P. Glorieux, Phys. Rev. E 49, R971 (1994).

[14] T. Pierre, G. Bonhomme, and A. Atipo, Phys. Rev. Lett. 76, 2290 (1996).

[15] K. Hall, D. J. Christini, M. Tremblay, J. J. Collins, L. Glass, and J. Billette, Phys. Rev. Lett. 78, 4518 (1997).

[16] D. W. Sukow, M. E. Bleich, D. J. Gauthier, and J. E. S. Socolar, Chaos 7, 560 (1997).

[17] P. Parmananda, R. Madrigal, M. Rivera, L. Nyikos, I. Z. Kiss, and V. Gáspár, Phys. Rev. E 59, 5266 (1999).

[18] E. Benkler, M. Kreuzer, R. Neubecker, and T. Tschudi, Phys. Rev. Lett. 84, 879 (2000).

[19] O. Lüthje, S. Wolff, and G. Pfister, Phys. Rev. Lett. 86, 1745 (2001).

[20] H. Benner and W. Just, J. Kor. Phys. Soc. 40, 1046 (2002).

[21] C. Beta, M. Bertram, A. S. Mikhailov, H. H. Rotermund, and G. Ertl, Phys. Rev. E 67, 046224 (2003).

[22] G. Franceschini, S. Bose, and E. Schöll, Phys. Rev. E 60, 5426 (1999).

[23] O. Beck, A. Amann, E. Schöll, J. E. S. Socolar, and W. Just, Phys. Rev. E 66, 016213 (2002).

[24] J. Unkelbach, A. Amann, W. Just, and E. Schöll, Phys. Rev. E 68, 026204 (2003).

[25] J. Schlesner, A. Amann, N. B. Janson, W. Just, and E. Schöll, Phys. Rev. E 68, 066208 (2003).

[26] C. Beta, A. S. Mikhailov, Controlling spatiotemporal chaos in oscillatory reaction-diffusion systems by time-delay autosynchronization, to appear in Physica D.

[27] N. Baba, A. Amann, E. Schöll, and W. Just, Phys. Rev. Lett. 89, 074101 (2002).

[28] W. Just, S. Popovich, A. Amann, N. Baba, and E. Schöll, Phys. Rev. E 67, 026222 (2003).

[29] W. Just, D. Reckwerth, J. Möckel, E. Reibold, and H. Benner, Phys. Rev. Lett 81, 562 (1998).

[30] G. Kociuba and N. R. Heckenberg, Phys. Rev. E 68, 066212 (2003).

[31] J. Xua, K.W. Chungb, Physica D 180 17-39 (2003).

[32] K. Engelborghs, T.Luzyanina, G. Samaey, DDE-BIFTOOL v. 2.00: a Matlab package for bifurcation analysis of delay differential equations, Technical Report TW-330, Department of Computer Science, K.U.Leuven, Leuven, Belgium, 2001; K. Engelborghs, T. Luzyanina and D. Roose, Numerical bifurcation analysis of delay differential equations using DDEBIFTOOL, ACM Transactions on Mathematical Software, Vol. 28, Number 1, p. 1-21, 2002. 